\title{
Neurophysiological evidence for transfer appropriate processing of memory: Processing versus feature similarity
}

\author{
HaLine E. SCHENDAN \\ Tufts University, Medford, Massachusetts \\ MGH-NMR Martinos Center, Charlestown, Massachusetts \\ and Boston University, Boston, Massachusetts \\ AND \\ Marta Kutas \\ University of California, San Diego, California
}

\begin{abstract}
Transfer appropriate processing (TAP) accounts propose that memory is a function of the degree to which the same neural processes transfer appropriately from the study experience to the memory test. However, in prior research, study and test stimuli were often similar physically. In two experiments, event-related brain potentials (ERPs) were recorded to fragmented objects during an indirect memory test to isolate transfer of a specific perceptual process from overlap of physical features between experiences. An occipitotemporoparietal P2(00) at 200 msec showed implicit memory effects only when similar perceptual grouping processes of good continuation were repeatedly engaged - despite physical feature differences - as TAP accounts hypothesize. This result provides direct neurophysiological evidence for the critical role of process transfer across experiences for memory.
\end{abstract}

Memory is best when retrieved under circumstances identical to the original experience. Transfer appropriate processing (TAP) accounts explain this finding by the proposal that memory is a function of the degree to which initial processing of an experience is appropriate for processing demands during the subsequent memory task (Morris, Bransford, \& Franks, 1977). Typically, tests of the TAP hypothesis manipulate task requirements from study to test, while keeping the stimuli identical (i.e., the same physical stimuli are subjected to either the same task at study and at test, or different tasks). Consistent findings of worse memory performance when tasks differ have been attributed to task-related processes. However, memory for studied items - regardless of task condition-may reflect transfer not just of all the processes the same physical stimulus first engages at study and then again at test, but also all the repeated physical stimulus representations. This raises a question, investigated herein, about the perceptual specificity of memory transfer. Must a stimulus remain physically identical for memory in a specific processing network to transfer from the study to the test experience?

Relevant evidence has come from studies on the perceptual specificity of memory and object constancy in the visual cognition field. Such studies typically isolate the contribution of the physical stimulus to memory by using the same task while manipulating the stimulus. Even so, few of these ex- periments were designed to address TAP, and most employed words - a special linguistic image class. Moreover, nearly all retained some physical overlap between study and test stimuli (for a review, see Bowers, 2000), thereby confounding transfer of perceptual processes with overlap of physical features, probably because teasing apart these factors was not their aim. Consequently, memory has been attributed to processing transfer (perhaps inappropriately) in neuroimaging (see, e.g., Prince, Daselaar, \& Cabeza, 2005) and some behavioral studies, even when features are reinstated at test (for a review, see Leboe, Whittlesea, \& Milliken, 2005; Tenpenny, 1995). For instance, in most object cognition studies relevant to ours, study and test pictures overlapped in higher order parts and local contours (Biederman \& Cooper, 1991; Schacter, Cooper, \& Treadwell, 1993; Schacter et al., 1997; Schendan \& Kutas, 2003; Snodgrass \& Feenan, 1990; Snodgrass, Hirshman, \& Fan, 1996; Srinivas, 1993). In three studies - including one using neuroimaging-local contour did not overlap, but because the focus was on shape representation, processing was not manipulated; stimulus and processing transfer thus remained entangled (Biederman \& Cooper, 1991; Kourtzi \& Kanwisher, 2001; Snodgrass et al., 1996). The question therefore remains whether the TAP emphasis on processing appropriateness between study and test for memory is wholly warranted, or whether physical feature overlap is as critical. 


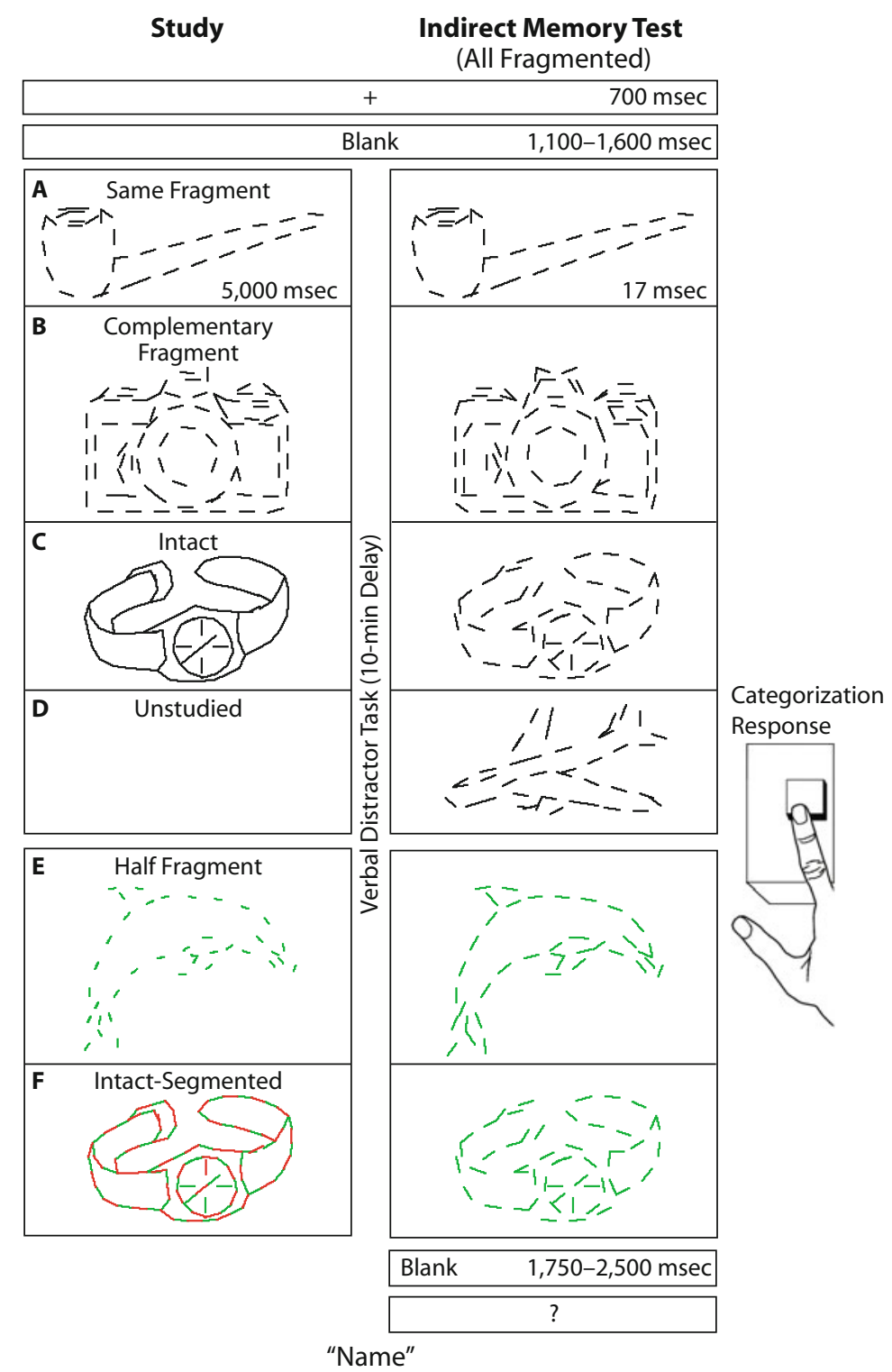

Figure 1. Methods for Experiments 1 and 2. In both experiments, participants categorized objects during a study phase followed by an indirect memory test. Throughout, a categorization response was indicated by a button press and then a spoken name. Guessing was encouraged; if participants had no idea, they withheld the categorization response and said "Don't know." During the test phase, all line drawings of objects were shown in fragmented form. A verbal distractor task ( 10 min) intervened between study and test. In the study phase, participants saw the (A) same fragments, in which identical stimuli appeared at both study and test, or the (B) complementary fragment, in which test objects shared no local features with the studied objects, because one picture had lines where its complementary fragment had gaps, and vice versa. (D) An unstudied condition had objects not shown previously in the study phase. (C) In Experiment 1, they also studied pictures that were intact, constructed by overlaying the two complementary fragments in black ink to create a picture with continuous lines. (E) In Experiment 2, participants also studied half fragments, in which studied objects were constructed with lines that were in the same location and orientation and half the length of those in the corresponding test object. (F) In Experiment 2, they also studied pictures that were like the intact ones in Experiment 1, but instead were intact-segmented, created by overlapping the two complementary fragmented versions of the object in different colors (i.e., red for one fragment and green for the other fragment that also appeared in the test). In Experiment 2, in the study phase, pictures in the same, complementary, and half conditions were drawn in green ink, and, in the test phase, all pictures were drawn in green ink. Note that the duration of each stimulus is shown; "Blank" denotes no stimulus; lines and gaps between them subtended $0.5^{\circ}$ of visual angle on the basis of neurophysiological studies of illusory contours and closure in monkey area V2 (Peterhans \& von der Heydt, 1989).

We designed two experiments to unconfound feature overlap and processing transfer and to assess their contributions to memory. In both experiments, participants categorized objects at the basic level (see, e.g., dog,
Rosch, Mervis, Gray, Johnson, \& Boyes-Braem, 1976) during both study and memory test phases. Long-term memory was assessed indirectly, because participants were not instructed explicitly to use memory for the prior 
Table 1

The Relationship Between the Stimuli at Study and at Test and the Predicted Pattern of ERP Repetition Effects (Studied vs. Unstudied Condition) Based on Each Hypothesis for Each of the Study Conditions in Experiments 1 and 2

\begin{tabular}{|c|c|c|c|c|}
\hline \multirow[b]{2}{*}{ Condition } & \multicolumn{2}{|c|}{ Study-Test Relationship } & \multicolumn{2}{|c|}{ Hypothesis } \\
\hline & $\begin{array}{c}\text { Grouping } \\
\text { Transfer }\end{array}$ & $\begin{array}{l}\text { Feature } \\
\text { Overlap }\end{array}$ & $\begin{array}{l}\text { Grouping } \\
\text { TAP }\end{array}$ & $\begin{array}{l}\text { Feature } \\
\text { Overlap }\end{array}$ \\
\hline \multicolumn{5}{|l|}{ Experiment 1} \\
\hline Same fragment & Full & Full & ++ & ++ \\
\hline Complementary fragment & Full & None & ++ & + or 0 \\
\hline Intact & None & Full & 0 & ++ \\
\hline Unstudied & None & None & 0 & 0 \\
\hline \multicolumn{5}{|l|}{ Experiment 2} \\
\hline Same fragment & Full & Full & ++ & ++ \\
\hline Half fragment & Full & Half & ++ & + or 0 \\
\hline Complementary fragment & Full & None & ++ & + or 0 \\
\hline Intact-Segmented & None & Full & 0 & ++ \\
\hline Unstudied & None & None & 0 & 0 \\
\hline
\end{tabular}

Note- “++" = repetition effect. "+" = reduced repetition effect. " 0 " = no repetition effect. "Full" means study and test stimuli both recruit and thus transfer grouping processes (grouping transfer column) or share exactly the same visual features (feature overlap column). "Half" means study and test stimuli share half of the same visual features (feature overlap column). "None" means study and test stimuli transfer no grouping processes (grouping transfer column) or share no visual features (feature overlap column).

study episode to aid performance in the test phase. Indirect memory tests are generally considered better suited than direct tests (i.e., recognition, recall) for characterizing perceptual processes and representations (Biederman \& Cooper, 1991). Repetition priming effects-better task performance with repeated rather than new itemsdepend upon perceptual systems in the occipitotemporal neocortex supporting implicit memory without conscious awareness, as opposed to the medial temporal lobe system supporting explicit recognition and recall with awareness (Tulving \& Schacter, 1990).

We sought evidence for TAP — despite no or partial stimulus feature overlap-and evidence of memory for features, despite no processing transfer. The stimulus features examined were line location, orientation, and length. Two perceptual grouping processes were investigated. (1) "Good continuation," which underlies the perception of multiple, smoothly continuing image features as one contour, and (2) "Closure," which underlies the perception of separate elements as a complete unitary region (Koffka, 1935).

These stimulus features and perceptual grouping processes were combined to yield four study-test conditions in Experiment 1 and five in Experiment 2 (Figure 1; Table 1). All test objects were presented as fragmented pictures. The study conditions ranged from full process transfer and full feature overlap (same fragment) to none (unstudied). Combinations in between had full processing transfer, but no feature overlap, or vice versa. In two conditions, studied objects were fragmented, leading to transfer of grouping processes, but the fragments differed from the test versions in local features, either all (complementary) or half (half, only Experiment 2); in two other conditions, maximal feature similarity (same contours) was retained between study and test objects, whereas grouping process transfer was minimized (if not eliminated), thereby assessing the processing specificity of the results (Experiment 1 intact; Experiment 2 intact-segmented).
According to a grouping TAP hypothesis, memory is better when the same neural processes of perceptual grouping that are engaged upon initial exposure (at study) are engaged again during subsequent experiences (at test), as opposed to when different component processes are recruited each time (Leboe et al., 2005; Tenpenny, 1995). This idea predicts (Table 1) that all study-test combinations that engage similar grouping processes should show repetition effects that pattern together (same, complementary, half), regardless of the degree of feature overlap, whereas no effects are predicted for conditions that do not engage these processes at study (intact[-segmented]). A feature overlap hypothesis predicts (Table 1) that conditions with overlapping features should show memory effects that pattern together, regardless of whether they engage grouping processes (same, intact[-segmented]), while conditions in which features differ (half, complementary) should show no or smaller memory effects. In short, although both hypotheses predict repetition effects for same fragments, the grouping TAP hypothesis predicts effects in both changed feature conditions (half, complementary), whereas the feature hypothesis predicts effects in both intact conditions (intact[-segmented]). Of course, features and processes may interact, predicting the largest repetition effects in the same condition, but some in all the others as well.

We recorded event-related potentials (ERPs) to complement response times (RTs). ERPs provide a direct, continuous measure of the neocortical activity supporting visual and memory processing, revealing memory as voltage differences between repeated and new items. Critically, different ERP components reflect distinct perceptualcognitive functions. We focused on memory-related effects on early sensory potentials - especially the occipitotemporoparietal P2(00) components that reflect posterior visual processing and vary with both visual stimulus features and perceptual grouping processes (Han, Jiang, Mao, 
Humphreys, \& Qin, 2005). Importantly, this P2 is more positive for attended, spatially separated, Gabor stimuli that are collinear, entailing good continuation to create perceived lines, in comparison with Gabor stimuli in an orthogonal orientation without good continuation (Khoe, Freeman, Woldorff, \& Mangun, 2006). P2 also precedes RT by several hundred milliseconds and is unlikely to be affected by later processes involved in object categorization, conceptual implicit memory, and explicit memory (Schendan \& Kutas, 2002, 2003; Voss \& Paller, 2006). Repetition effects on this early ERP would be harder to attribute to contamination by explicit memory, a prime concern in related behavioral (Bowers, 2000) and ERP (Voss \& Paller, 2006) studies. The P2 thus could better isolate neural activity related to perceptual implicit memory for good continuation and closure computations.

\section{EXPERIMENT 1}

\section{Method}

Design. A $2 \times 2$ repeated measures factorial design (Table 1) used variables of grouping (full, none) and feature (full, none).

Materials. In-house software generated complementary fragmented and intact versions of 272 simplified line drawings of objects from Snodgrass and Vanderwart (1980) or copyright-free sources (Figures 1A-1D). Four lists of 68 objects were matched on normative data (Snodgrass \& Corwin, 1988; Snodgrass \& Vanderwart, 1980) and categories (e.g., "mammal"). Each fragment and its complement were designated "odd" and "even." Both complementary fragments were overlaid to create intact drawings with continuous lines. All lists and odd/even fragments were fully counterbalanced across conditions between subjects. Objects were white against a black background on a monitor that was $62 \mathrm{~cm}$ away, yielding $0.5^{\circ}$ line segments (Peterhans \& von der Heydt, 1989).

Procedure. All study- and test-phase trials started with central fixation for $700 \mathrm{msec}$ followed 1,100-1,600 msec later by an object (Figure 1). Participants pressed a key (dominant hand) as soon as they categorized the object (categorization response). At test, all pictures were shown fragmented for $17 \mathrm{msec}$. At study, for $5 \mathrm{sec}$ each, participants saw the same or the complementary fragment or intact drawings. An unstudied condition had objects that were not shown in the study phase. At study and test, conditions were randomly intermixed. Participants remained motionless from fixation until $2 \mathrm{sec}$ after the object appeared at study or, at test, until a cue "?" appeared 1,750-2,500 msec after the object disappeared. Participants named the object after the picture disappeared at study or after the cue appeared at test. Before each phase, they practiced with nonexperimental objects.

Electroencephalography (EEG). EEG was sampled at $250 \mathrm{~Hz}$ (bandpass filtered $0.01-100 \mathrm{~Hz}$ ) from 28 tin electrodes on a plastic cap. Cap and right mastoid electrodes and one below the right eye (monitoring blinks) were referenced to the left mastoid. Bilateral eye electrodes (monitoring saccades) were referenced to each other. ERPs were calculated by averaging EEG in each condition, excluding trials with above threshold muscle activity, blinks, eye and other movement artifacts, time-locking to image onset with a $150-\mathrm{msec}$ prestimulus baseline, and rereferencing to the mean of both mastoids.

Participants. Undergraduates volunteered for course credit and/ or cash. Data from 13 participants were excluded for excessive artifacts, leaving 32 participants: $18-28$ years old $(\mu=20), 17$ women.

Analyses. Test trials for objects categorized correctly in both phases-test and study (eliminating 6\% incorrect) — were analyzed, since priming is found primarily after spontaneously correct categorization (Snodgrass \& Feenan, 1990). Categorization was correct if participants reported names that were from Snodgrass and
Vanderwart (1980) or that were spoken in response to intact versions, synonyms, or synonymous nonperceptual descriptions. Mixed ANOVAs included between-subjects factors of list and odd-even fragment. For ERPs, mean amplitudes of a P1(00) (80-140 msec) and P2(00) (200-300 msec) were measured. ANOVAs had a withinsubjects factor of electrode. One ANOVA covered midline (labeled) sites 1, 26, 23, 6; another ANOVA covered 12 lateral electrode pairs (no label) to include a within-subjects factor of hemisphere (left, right). Huynh-Feldt adjustments to degrees of freedom corrected for sphericity violations.

\section{RESULTS}

\section{Memory Test Performance}

Omnibus ANOVAs on RTs showed a significant study main effect (same, complementary, intact, unstudied) $[F(3,72)=41.69, p<.0001]$ (Figure 2C). Planned comparisons demonstrated that RTs were faster in comparison with unstudied objects-in same fragment $[F(1,24)=$ 53.63], complementary fragment $(F=67.78)$, and intact conditions $(F=44.02)(p s<.0001)$. RTs were faster for same than for complementary fragments $(F=4.91, p<$ $.04)$ or intact conditions $(F=12.53, p<.005)$; intact and complementary conditions did not differ from each other $(p>9)$. Categorization accuracy was high (same and complementary, both $82 \%$; intact, $91 \%$; unstudied, $74 \%) .{ }^{1}$

\section{Memory Test ERPs}

P2(00). Repetition modulated the P2(00) over occipitotemporoparietal sites. This $\mathrm{P} 200$ repetition effect was specific to objects that were studied in fragmented form, as the grouping TAP hypothesis predicts: In comparison with those for unstudied objects, P200s in both the same and complementary conditions were smaller (i.e., less positive), whereas those for intact objects were not (Figures 2A and 2B). The omnibus ANOVAs included within-subjects factors of grouping and feature. Neither main effects of feature nor feature $\times$ grouping interactions were significant $(F \mathrm{~s}<1.5, p \mathrm{~s}>.2)$. However, grouping $\times$ electrode interactions were significant $[F(11,264)=7.19, p=$ $.0016, \varepsilon=.3180$; midline $F(3,72)=3.56, p=.0358, \varepsilon=$ .7055] (main electrode effect, $F=12.08, p=.0001, \varepsilon=$ .2014). ANOVAs on electrode pairs in 12 -msec periods around the P200 peak for the same and unstudied contrast localized the effect to occipitotemporoparietal locations (Table 2).

Planned pairwise comparisons were also conducted. Hochberg's (1988) step-up Bonferroni procedure set acceptable $p$ values $(p=.01$; midline $p=.0083)$. To test for repetition effects, each studied condition was compared with the unstudied condition: Condition $\times$ electrode interactions were significant for same $[F(11,264)=5.04$, $p=.0031, \varepsilon=.2805]$ and complementary $[F=5.60, p=$ $.0005, \varepsilon=.3342$; marginal midline $F(3,72)=4.20, p=$ $.0089, \varepsilon=.8864$ ] fragments, but not the intact condition $(F \mathrm{~s}<2, p \mathrm{~s}>.2)$. Comparisons were also made among the studied conditions: Condition $\times$ electrode interactions were marginal between same fragments versus intact $(F=$ 2.17, $p=.0986, \varepsilon=.2981$; midline $F=2.37, p=.0784$, $\varepsilon=.8911)$ and complementary fragments versus intact $(F=2.45, p=.0699, \varepsilon=.2862$; midline $F=3.97, p=$ 
A

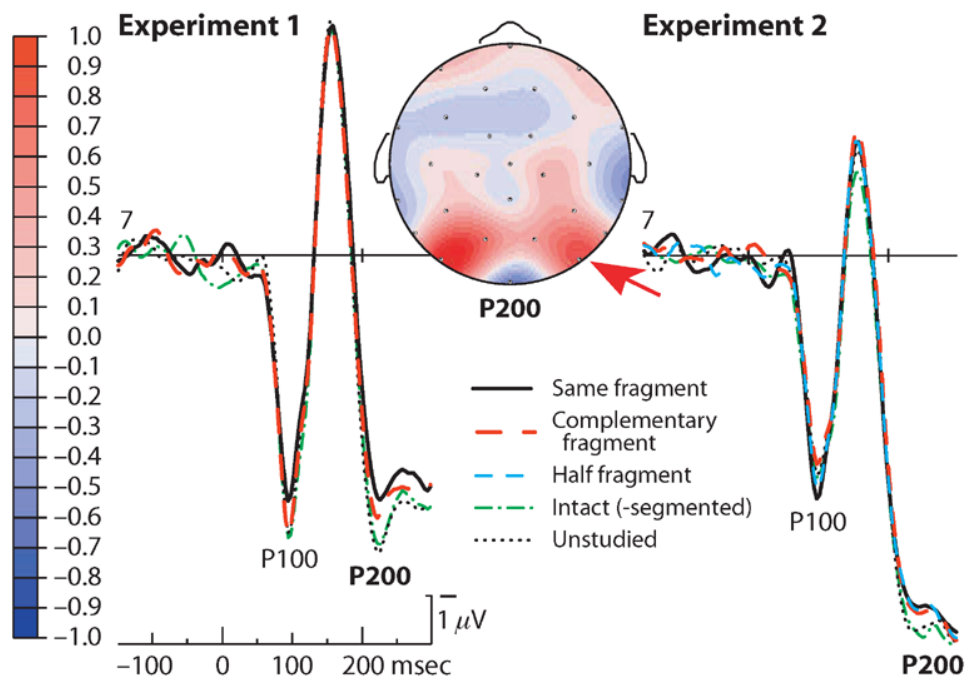

B

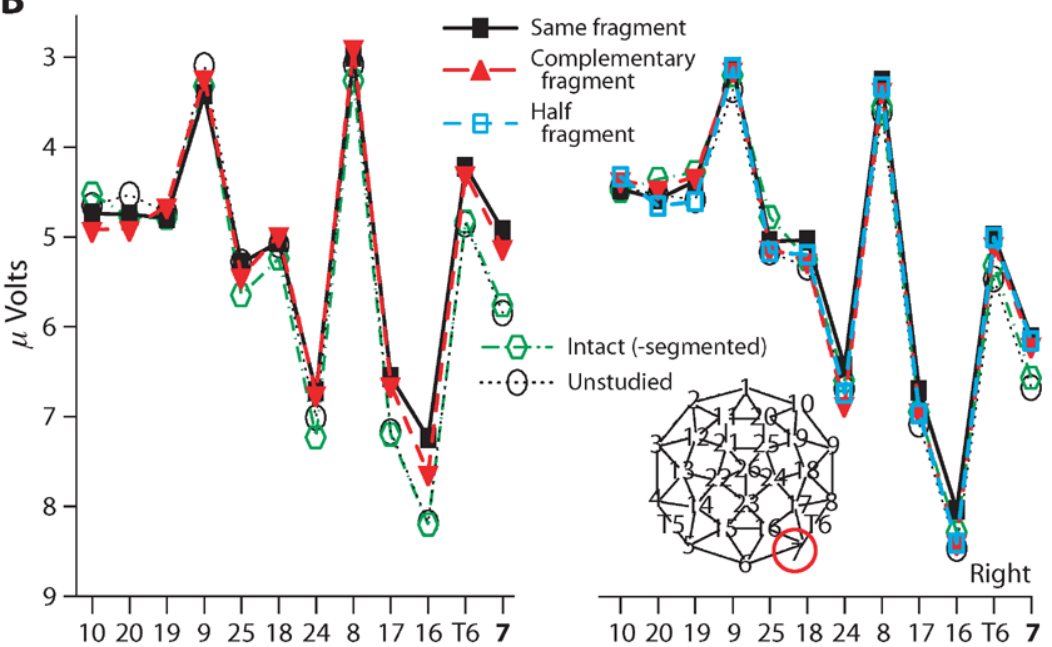

C
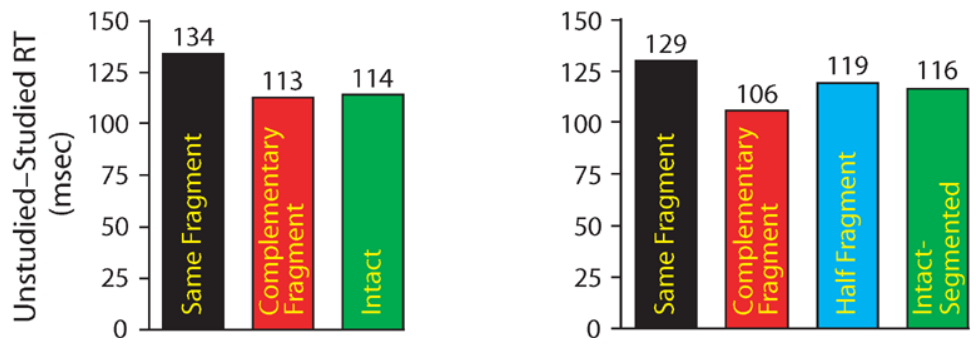

Figure 2. Grand averaged P200s and RT priming effects during the indirect memory test in Experiments 1 and 2 . (A) P200 repetition effects in both experiments are shown at the right occipitotemporal electrode 7 between -150 and 250 msec; ERPs are plotted with negative voltage up. In Experiment 1, P200s in same and complementary conditions were reduced in comparison with intact and unstudied conditions. In Experiment 2, P200s in same, complementary, and half conditions were reduced in comparison with intact-segmented and unstudied conditions. In between the ERP plots, a current source density (CSD) map (Schendan \& Kutas, 2003) shows the distribution across the head of current sources (red) and sinks (blue) for the P200 (226-238 msec). Note that the unstudied condition in Experiment 1 is shown as a representative example; the normalized current scale is shown (left); the arrow shows the location of right occipitotemporal electrode site 7. (B) Line graphs show P200 amplitude across right lateral sites between 224 and 240 msec in both experiments. The $\mathbf{P 2 0 0}$ repetition effect was largest over the right hemisphere, particularly over occipitotemporal sites T6 and 7 (circled on montage). The geodesic electrode montage used to record EEG is shown between the graphs; note that T5, T6, $26(\mathrm{Cz})$ and $6(\mathrm{Oz})$ were from the 10-20 system. (C) In Experiment 1 (left bar plot), categorization RTs showed significant priming (unstudied-studied) in all studied conditions. RTs were fastest in the same condition (670 msec; black bars), intermediate in complementary (691 msec; red bars) and intact (690 msec; green bars) conditions, and slowest for unstudied objects (804 msec). In Experiment 2 (right bar plot), RTs showed priming (unstudied-studied) in all conditions. RTs were fastest in same (679 msec), half (690 msec; blue bar), and intact-segmented (692 msec) conditions, intermediate for complementary fragments (703 msec), and slowest for unstudied objects $(809$ msec). 
Table 2

Occipitotemporoparietal Scalp Distribution of P200 Repetition Effects: Pairwise Electrode Sites Showing Significant Repetition Effects in ANOVAs for Contrasts of Same Versus Unstudied Fragments in Experiment 1

\begin{tabular}{lccccccc}
\hline & \multicolumn{7}{c}{ Time $(\mathrm{msec})$} \\
\cline { 2 - 8 } Sites & $178-190$ & $194-206$ & $210-222$ & $226-238$ & $242-254$ & $258-270$ & $274-286$ \\
\hline 3,9 & n.s. & n.s. & n.s. & n.s. & $\mathrm{S}^{*}$ & $\mathrm{~S}^{*}$ & n.s. \\
4,8 & n.s. & n.s. & $\mathrm{S}^{*}$ & n.s. & n.s. & n.s. & n.s. \\
14,17 & n.s. & $\mathrm{S}^{*}$ & $\mathrm{~S}^{*}$ & n.s. & n.s. & n.s. & n.s. \\
T5,T6 & n.s. & $\mathrm{S}^{*}$ & $\mathrm{~S}^{* *}$ & $\mathrm{~S}^{*}$ & n.s. & n.s. & $\mathrm{S} \times \mathrm{H}^{* *}$ \\
15,16 & $\mathrm{~S}^{*}$ & $\mathrm{~S}^{* *}$ & $\mathrm{~S}^{* *}$ & $\mathrm{~S}^{*}$ & n.s. & n.s. & n.s. \\
5,7 & $\mathrm{~S}^{*}$ & $\mathrm{~S}^{* *}$ & $\mathrm{~S}^{* * *}$ & $\mathrm{~S}^{* *}$ & $\mathrm{~S}^{*}$ & n.s. & n.s. \\
23,6 & $\mathrm{~S}^{*}$ & $\mathrm{~S}^{* *}$ & $\mathrm{~S}^{* *}$ & $\mathrm{~S}^{*}$ & $\mathrm{~S}^{*}$ & $\mathrm{~S} \times \mathrm{E}^{*}$ & $\mathrm{~S} \times \mathrm{E}^{* *}$ \\
\hline
\end{tabular}

Note- $d f=1,24$. S, study; $\mathrm{S} \times \mathrm{E}$, study by electrode interaction; $\mathrm{S} \times \mathrm{H}$, study by hemisphere interaction. ${ }^{*} p<.05 . \quad{ }^{* *} p<.01 . \quad{ }^{* * *} p<.001$.

$.0242, \varepsilon=.7999)$, but not same versus complementary $(F \mathrm{~s}<1, p \mathrm{~s}>.4)$ fragments.

P1(00). No grouping or feature main effects or interactions were significant $(F \mathrm{~s}<1.8, p \mathrm{~s}>.19)$.

\section{EXPERIMENT 2}

\section{Method}

Experiment 1 methods were used, except for the following.

Design. The intact-segmented condition replaced the intact one. The half condition was added (Figures 1A-1B, 1D-1F).

Materials. Stimuli included 68 more copyright-free objects. Five lists were matched to each other and to Experiment 1 lists. For half-segment versions of all fragmented pictures, the length of each line was reduced by $50 \%$. For intact-segmented pictures, one fragment was colored green and overlaid with its complement colored in red, and matched for luminance $(Y, x, y)$ : green (13.7, $0.282,0.542)$, red $(13.8,0.516,0.285)$. All fragmented pictures were green. The monitor was located $93 \mathrm{~cm}$ away from the participant's eyes, yielding $0.5^{\circ}$ lines and gaps.

Participants. Data from 8 participants were excluded, leaving 40 participants: $18-28$ years old $(\mu=20.5) ; 24$ female.

Analyses. Omnibus ANOVAs had a study factor (same, complementary, half, intact-segmented, unstudied).

\section{RESULTS}

\section{Memory Test Performance}

The omnibus ANOVA on RTs (Figure 2C) showed a significant main effect of study $[F(4,120)=49.14, p<$ $.0001]$. Planned comparisons showed RTs were faster than unstudied objects in all studied conditions $[F \mathrm{~s}(1,30)>$ $19, p s<.001]$. RTs in the same condition were significantly faster than those in the complementary condition $[F=10.06, p=.0035]$, but not in the half and intactsegmented conditions $(F \mathrm{~s}<2.3, p \mathrm{~s}>.14)$. Accuracy was good: same $(73 \%)$, complementary $(72 \%)$, half $(65 \%)$, intact-segmented (85\%), unstudied (66\%). (See note 1.)

\section{Memory Test ERPs}

P2(00). P200 amplitude was modulated, although mainly over the right hemisphere (Figures 2A and 2B). The omnibus ANOVAs showed a significant study $\times$ hemisphere interaction $[F(4,120)=3.04, p=.0198]$ [main electrode effects, $F(11,330)=30.58, \varepsilon=.2146$, midline $F(3,90)=8.95, \varepsilon=.7106, p s<.0005]$.
Planned pairwise ANOVAs further tested the hypotheses with a primary focus on the two newly added half and intact-segmented conditions. The grouping TAP hypothesis predicts memory transfer (i.e., P200 repetition effects) that is comparable for both same and half fragment conditions, and no memory transfer for the intact-segmented condition, which did not engage grouping processes at study. By contrast, the feature hypothesis (or a hybrid grouping TAP and feature idea) predicts larger memory effects for same than half, but comparable effects for the same fragment and intact-segmented conditions. Comparing the unstudied condition with each study condition, in turn, revealed that study $\times$ hemisphere interactions were significant for the same $[F(1,30)=4.19, p=.0495]$ and half $(F=4.85, p=.0354)$ fragments, and were marginal for the complementary fragments $(F=3.10, p=$ $.0884)$, but were not reliable for intact-segmented $(F=$ $0.02, p=.8993)$. Comparisons among the study conditions revealed that the study $\times$ hemisphere interactions were significant for half versus intact-segmented $(F=$ 4.94, $p=.0339)$, and same versus intact-segmented $(F=$ 9.39, $p=.0046)$, but not for same versus half $(F \mathrm{~s}<1$, $p s>.4)$; complementary fragments differed marginally from intact-segmented $(F=3.82, p=.06)$, but not from same or half fragments $(F \mathrm{~s}<0.6, p \mathrm{~s}>.46)$. Thus, $\mathrm{P} 200$ repetition effects were similarly observed in same, complementary (marginal), and half fragment conditions, but not in the intact-segmented condition, supporting only the grouping TAP hypothesis.

P1(00). There were no main effects or interactions thereof in omnibus $(F \mathrm{~s}<1.1, p \mathrm{~s}>.39)$ or comparison ANOVAs $(F \mathrm{~s}<2.6, p \mathrm{~s}>.11)$.

\section{DISCUSSION}

Findings from two ERP experiments provide direct neurophysiological evidence that the appropriate transfer of a perceptual process between study and test experiences can be an important determinant of early memory reactivation (related to repetition priming of objects), despite differences in lower level visual features between exposures. The pattern of memory-related modulation of early neocortical activity around $200-300 \mathrm{msec}$ is consistent with the perceptual grouping TAP hypothesis and provides no 
support for the feature hypothesis. The reduction in the amplitude of an occipitotemporoparietal P2(00) component for repeated objects in comparison with new ones seems to depend on whether the same processes of good continuation and closure are recruited during both study and test experiences: It occurred only when both study and test items were fragmented somehow and thus recruited perceptual grouping processes. Moreover, the P200 was reduced to a similar extent whether the study-test features were identical (same), different in length (half), or different entirely, being at other spatial locations and orientations (complementary). Reinstantiation of the physical features from study to test thus is neither necessary nor sufficient for this grouping TAP benefit of memory reflected in the posterior P200. Despite substantial feature overlap, no P200 repetition effects were observed for objects studied initially as intact images, whether drawn using a continuous contour (intact, Experiment 1) or with contiguous line segments of alternating colors (intactsegmented, Experiment 2). Indeed, the P200s to intact pictures most resemble those to objects that were never studied (Schendan \& Kutas, 2003). All intact and unstudied conditions had larger P200s than those to objects studied in fragmented images, whether the fragments were the same, complementary, or half sized.

Our characterization of the $\mathrm{P} 200$ repetition effect as reflecting memory for good continuation processes is consistent with prior findings. A 190-270-msec time course for perceptual organization processes in occipitotemporal neocortex has been indicated in behavioral (Biederman, 1987; Sekuler \& Palmer, 1992) and neuroimaging investigations (Halgren, Mendola, Chong, \& Dale, 2003; Mendola, Dale, Fischl, Liu, \& Tootell, 1999). As we also observed, P2(00) grouping-related modulations have a right lateral occipitotemporoparietal distribution (Han et al., 2005). Notably, P200 effects happen after the earliest stage of perceptual categorization, as indexed by the frontocentral P150 (Schendan, Ganis, \& Kutas, 1998), leaving open the possibility that lower order object knowledge influences these perceptual grouping operations (Peterson \& Gibson, 1994). One possibility is that visual knowledge directs attention to salient image features. P2(00) grouping effects are known to be influenced by attention, task, and implicit learning manipulations (Han et al., 2005; Khoe et al., 2006; Song et al., 2005).

The P200 repetition effect occurs well before 300 $600 \mathrm{msec}$ - the time period associated with conceptual implicit memory and explicit memory (Voss \& Paller, 2006), supporting a key timing prediction of multiple memory systems theories (Tulving \& Schacter, 1990). This timing suggests that the P200 repetition effects emanate from cortical areas that support perceptual implicit memory and that are activated before explicit recollection.

By contrast, priming of RTs and later ERPs in both experiments (see Schendan \& Kutas, 2007) suggest that the impact of feature overlap on memory-related processes occurs after $300 \mathrm{msec}$. All studied objects show some RT priming, with the most for same fragments, as was previously reported for objects spontaneously categorized correctly (Snodgrass et al., 1996), as were those herein.
This dissociation between the RT and P200 measures is not surprising, given that RTs reflect the cumulative effect of memory-related effects on perceptual, cognitive, and motor responses (see Schendan \& Kutas, 2003; Vogel, Luck, \& Shapiro, 1998), and not just on the perceptual processes that the $\mathrm{P} 200$ reflects.

In sum, by using fragmented and intact pictures at study and fragmented pictures at test, in combination with an ERP measure sensitive to perceptual grouping, we were able to tease apart the contribution of perceptual processing versus feature similarity to memory. The pattern of occipitotemporoparietal P2(00) findings in two experiments demonstrates that transfer of implicit memory for processing related to perceptual grouping can occur, despite changes in local features between experiences. Perhaps surprisingly, perceptual features appear to be important mainly insofar as they determine how stimuli are processed. These findings provide direct neurophysiological support for the critical role of neural processing similarity across experiences for the quality of memory, as hypothesized by TAP.

\section{AUTHOR NOTE}

This work was supported by Tufts University faculty research funds and an FRAC Research Semester Fellowship and Grant NS052914 to H.E.S., and Grants MH52893 and AG05914 to M.K., who was a Lady Davis Fellow during the writing of this manuscript. The authors thank Elizabeth Hammonds at Tufts University for assistance analyzing the performance data from Experiment 2. Address correspondence to H. E. Schendan, Department of Psychology, Tufts University, The Psychology Building, 490 Boston Ave., Medford, MA 02155 (e-mail: haline_e .schendan@tufts.edu).

\section{REFERENCES}

Biederman, I. (1987). Recognition-by-components: A theory of human image understanding. Psychological Review, 94, 115-117.

Biederman, I., \& Cooper, E. E. (1991). Priming contour-deleted images: Evidence for intermediate representations in visual object recognition. Cognitive Psychology, 23, 393-419.

BOWERS, J. S. (2000). In defense of abstractionist theories of repetition priming and word identification. Psychonomic Bulletin \& Review, 7, 83-99.

Halgren, E., Mendola, J., Chong, C. D., \& Dale, A. M. (2003). Cortical activation to illusory shapes as measured with magnetoencephalography. NeuroImage, 18, 1001-1009.

Han, S., Jiang, Y., MaO, L., HumphreYs, G. W., \& Qin, J. (2005). Attentional modulation of perceptual grouping in human visual cortex: ERP studies. Human Brain Mapping, 26, 199-209.

HochberG, Y. (1988). A sharper Bonferroni procedure for multiple tests of significance. Biometrika, 75, 800-802.

Khoe, W., Freeman, E., WoldorfF, M. G., \& Mangun, G. R. (2006). Interactions between attention and perceptual grouping in human visual cortex. Brain Research, 1078, 101-111.

KoffKa, K. (1935). Principles of Gestalt psychology. New York: Harcourt, Brace and Company.

KourtzI, Z., \& Kanwisher, N. (2001). Representation of perceived object shape by the human lateral occipital complex. Science, 293, 1506-1509.

Leboe, J. P., Whittlesea, B. W., \& Milliken, B. (2005). Selective and nonselective transfer: Positive and negative priming in a multipletask environment. Journal of Experimental Psychology: Learning, Memory, \& Cognition, 31, 1001-1029.

Mendola, J. D., Dale, A. M., Fischl, B., Liu, A. K., \& Tootell, R. B. (1999). The representation of illusory and real contours in human cortical visual areas revealed by functional magnetic resonance imaging. Journal of Neuroscience, 19, 8560-8572.

Morris, C. D., BRANSFORD, J. D., \& Franks, J. J. (1977). Levels of 
processing versus transfer appropriate processing. Journal of Verbal Learning \& Verbal Behavior, 16, 519-533.

PETERHANS, E., \& VON DER HEYDT, R. (1989). Mechanisms of contour perception in monkey visual cortex. II. Contours bridging gaps. Journal of Neuroscience, 9, 1749-1763.

Peterson, M. A., \& GibSON, B. S. (1994). Object recognition contributions to figure-ground organization: Operations on outlines and subjective contours. Perception \& Psychophysics, 56, 551-564.

Prince, S. E., DaselaAr, S. M., \& Cabeza, R. (2005). Neural correlates of relational memory: Successful encoding and retrieval of semantic and perceptual associations. Journal of Neuroscience, $\mathbf{2 5}$, 1203-1210.

Rosch, E., Mervis, C., Gray, W., Johnson, D., \& Boyes-Braem, P. (1976). Basic objects in natural categories. Cognitive Psychology, $\mathbf{8}$, 382-439.

Schacter, D. L., Cooper, L. A., \& Treadwell, J. (1993). Preserved priming of novel objects across size transformation in amnesic patients. Psychological Science, 4, 331-335.

Schacter, D. L., Uecker, A., Reiman, E., Yun, L. S., Bandy, D., Chen, K., ET AL. (1997). Effects of size and orientation change on hippocampal activation during episodic recognition: A PET study. NeuroReport, 8, 3993-3998.

Schendan, H. E., Ganis, G., \& Kutas, M. (1998). Neurophysiological evidence for visual perceptual categorization of words and faces within 150 msec. Psychophysiology, 35, 240-251.

Schendan, H. E., \& Kutas, M. (2003). Time course of processes and representations supporting visual object identification and memory. Journal of Cognitive Neuroscience, 15, 111-135.

ScHENDAN, H. E., \& KUTAS, M. (2007). Neurophysiological evidence for the timecourse of activation of global shape, part, and local contour representations during visual object categorization and memory. Journal of Cognitive Neuroscience, 19, 734-749.

SeKuler, A. B., \& Palmer, S. E. (1992). Perception of partly occluded objects: A microgenetic analysis. Journal of Experimental Psychology: General, 121, 95-111.

SNODGRASS, J. G., \& CORWIN, J. (1988). Perceptual identification thresholds for 150 fragmented pictures from the Snodgrass and Vanderwart picture set. Perceptual \& Motor Skills, 67, 3-36.
Snodgrass, J. G., \& Feenan, K. (1990). Priming effects in picture fragment completion: Support for the perceptual closure hypothesis. Journal of Experimental Psychology: General, 119, 276-296.

SNODGRASS, J. G., HiRSHMAN, E., \& FAN, J. (1996). The sensory match effect in recognition memory: Perceptual fluency or episodic trace? Memory \& Cognition, 24, 367-383.

SNODGRASS, J. G., \& VANDERWART, M. (1980). A standardized set of 260 pictures: Norms for name agreement, image agreement, familiarity, and visual complexity. Journal of Experimental Psychology: Human Learning \& Memory, 6, 174-215.

Song, Y., Ding, Y., FAN, S., QU, Z., XU, L., Lu, C., ET AL. (2005). Neural substrates of visual perceptual learning of simple and complex stimuli. Clinical Neurophysiology, 116, 632-639.

SRINIVAS, K. (1993). Perceptual specificity in nonverbal priming. Journal of Experimental Psychology: Learning, Memory, \& Cognition, 19, 582-602.

TENPENNY, P. L. (1995). Abstractionist versus episodic theories of repetition priming and word identification. Psychonomic Bulletin \& Review, 2, 339-363.

Tulving, E., \& Schacter, D. L. (1990). Priming and human memory systems. Science, 247, 301-306.

VoGel, E. K., LUCK, S. J., \& SHAPIRO, K. L. (1998). Electrophysiological evidence for a postperceptual locus of suppression during the attentional blink. Journal of Experimental Psychology: Human Perception \& Performance, 24, 1656-1674.

Voss, J. L., \& PALleR, K. A. (2006). Fluent conceptual processing and explicit memory for faces are electrophysiologically distinct. Journal of Neuroscience, 26, 926-933.

\section{NOTE}

1. In both experiments, although statistics indicated test accuracy results reflected better accuracy at study for intact than fragmented pictures $(F \mathrm{~s}>46, p \mathrm{~s}<.0001)$, this cannot explain the RT or P200 results, which showed a different pattern.

(Manuscript received January 24, 2006; revision accepted for publication September 11, 2006.) 